\title{
Out-of-focus holography at the Green Bank Telescope
}

\author{
B. Nikolic ${ }^{1,2}$, R. M. Prestage ${ }^{1}$, D. S. Balser ${ }^{1}$, C. J. Chandler ${ }^{3}$, and R. E. Hills ${ }^{4}$ \\ 1 National Radio Astronomy Observatory, PO Box 2, Green Bank, WV 24944, USA \\ 2 National Radio Astronomy Observatory, 520 Edgemont Road, Charlottesville, Va 22903, USA \\ e-mail: bnikolic@nrao.edu \\ 3 National Radio Astronomy Observatory, PO Box 0, Socorro, NM 87801, USA \\ ${ }^{4}$ Mullard Radio Astronomy Observatory, Cavendish Laboratory, Cambridge CB3 OHE, UK
}

Received 6 June 2006 / Accepted 4 January 2007

\section{ABSTRACT}

\begin{abstract}
We describe phase-retrieval holography measurements of the 100-m diameter Green Bank Telescope using astronomical sources and an astronomical receiver operating at a wavelength of $7 \mathrm{~mm}$. We use the technique with parameterization of the aperture in terms of Zernike polynomials and employing a large defocus, as described by Nikolic et al. (2007, A\&A, 465, 679). Individual measurements take around $25 \mathrm{~min}$ and from the resulting beam maps (which have peak signal to noise ratios of 200:1) we show that it is possible to produce low-resolution maps of the wavefront errors with accuracy around $\lambda / 100$.

Using such measurements over a wide range of elevations, we have calculated a model for the wavefront-errors due to the uncompensated gravitational deformation of the telescope. This model produces a significant improvement at low elevations, where these errors are expected to be the largest; after applying the model, the aperture efficiency is largely independent of elevation. We have also demonstrated that the technique can be used to measure and largely correct for thermal deformations of the antenna, which often exceed the uncompensated gravitational deformations during daytime observing.

We conclude that the aberrations induced by gravity and thermal effects are large-scale and the technique used here is particularly suitable for measuring such deformations in large millimetre wave radio telescopes.
\end{abstract}

Key words. telescopes

\section{Introduction}

The performance of large single-dish radio telescopes operating at millimetre and sub-millimetre wavelengths is often limited by the accuracy and alignment of the optical elements. These inaccuracies may arise during manufacture, assembly and adjustment, or they could be due to uncompensated gravitational deformation, or due to external factors such as differential heating or cooling of the structure. A commonly used method to measure such deformations using microwaves is known as "phaseretrieval holography" (see e.g. Morris 1985; Anderson \& Sali 1985). In this approach, only the amplitude of the antenna pattern is measured, and the phase of the aperture field (reflecting the deviations in the surface, and mis-collimation) is recovered by numerical processing. In this paper we describe the application of a new phase-retrieval holography technique, discussed in detail by Nikolic et al. (2007), to measure and correct such inaccuracies at the 100-m diameter Robert C. Byrd Green Bank Telescope (the GBT, see e.g., Jewell \& Prestage 2004).

The GBT has a very capable active surface control system (Lacasse 1998). The primary reflector is composed of 2004 panels mounted in rings that are concentric to the vertex of the $208 \mathrm{~m}$ parent (virtual) parabola. The surface accuracy of individual panels is about $75 \mu \mathrm{m}$. The panels are mounted at their corners on computer-controlled actuators such that the corners of four adjacent panels share one actuator; the actuators can be positioned to within a tolerance of $25 \mu \mathrm{m}$. The requirement is then to provide appropriate commands to the active surface control system to correct for the inaccuracies described above. Our long-term goal is to deliver a surface accuracy of $\sim 200 \mu \mathrm{m}$, suitable for telescope operation up to $115 \mathrm{GHz}$. Our intermediate goal, to allow operation up to $\sim 50 \mathrm{GHz}$, has a corresponding surface accuracy requirement of $360 \mu \mathrm{m}$ independent of elevation. Currently, the active surface is operated open-loop, using a simple look-up table of corrections as a function of elevation. Initially, these corrections were derived from a finite element model of the antenna. Although this provided a modest improvement over the static (photogrammetry) zero-point correction, there remained a significant fall-off in surface accuracy at low and high elevations. The need to provide an improved look-up table to correct for the unmodelled gravitational errors, and potentially also to correct for thermal effects, provided the motivation for the work described in this paper.

By using a parametrisation scheme employing Zernike polynomials and by making maps with a large defocus, the new measurement technique - which we colloquially refer to as Out-Of-Focus (OOF) holography - requires a significantly smaller dynamic range than "traditional" phase-retrieval holography measurements and hence can make use of astronomical sources and receivers. This in turn allows measurements to be made at a wide range of telescope elevations. Also, using the facility astronomical receivers means that each measurement provides a map of wavefront errors for the complete system used for astronomical observations, i.e., the combination of errors arising from surface inaccuracies, any mis-collimation and the phase response of the receiver.

The smaller required dynamic range is, however, achieved through a trade-off in the possible resolution in the map of wavefront errors (or, aberrations): typically we describe the wavefront errors as a linear combination of $20-30$ basis vectors. This means 
that OOF holography measurements are only sensitive to wavefront errors which are correlated over large scales in the aperture plane - in the case of the GBT, these spatial scales are around $20 \mathrm{~m}$ or larger.

This low resolution makes OOF holography unsuitable for panel-to-panel adjustment of large radio telescopes and for assessing the quality of individual panels. The ability to make measurements at a wide range of elevations, however, makes it possible to measure aberrations induced by gravitational deformations (Sect. 5). The ability to make measurements (comparatively) quickly, using the standard astronomical receivers with no special hardware setup, means that the technique can also potentially be used to measure and correct for non-repeatable effects, specifically thermal deformations, in "real-time".

\subsection{GBT surface setting and classes of deformations/corrections}

The GBT is designed to operate over a wide range of wavelengths, from approximately one meter $(300 \mathrm{MHz})$, through intermediate centimeter wavelengths, in to the millimeter regime ( $3 \mathrm{~mm}$ or $100 \mathrm{GHz}$ ). At the longest wavelengths, the antenna optics are essentially perfect. A number of successively more precise corrections are required to allow the antenna to operate effectively at the shorter wavelengths.

Prior to this work, the first three types of corrections described below were routinely being applied. Development of the fourth type of correction is a direct outcome of the work described here.

\subsubsection{Construction misalignment correction at the "rigging angle"}

During the construction of the GBT, one retro-reflector was placed on one of the panel corners adjacent to each actuator, and the locations of all 2004 retro-reflectors were measured using photogrammetry. Actuator zero-points were then derived which, as far as possible given the accuracy of the photogrammetry, compensate for actuator misalignments during the construction process. These settings were chosen so that the primary surface would be correct at the rigging angle of 50.3 degrees. The typical actuator zero-point correction is $1.9 \mathrm{~mm}$, and the root-meansquare (rms) panel to panel surface error after this correction is expected (from the accuracy of the photogrammetry) to be approximately $250 \mu \mathrm{m}$.

\subsubsection{Homologous deformation and subreflector "focus tracking"}

Like all modern large radio telescopes, the GBT is built to a homologous design (von Hoerner 1967). As the antenna tips from the zenith to the horizon, the primary mirror deforms due to the effect of gravity, but in such a way that the antenna remains (approximately) a parabola, and that the main effect of the deformation is simply to change the focal length of the parabola. At the same time, the offset feed-arm, which supports the sub-reflector and the receiver cabin at the Gregorian focus, also deflects under gravity. All of these effects are compensated for via a lookup table of sub-reflector lateral and radial focus translations as a function of elevation, which refocus the antenna to maximise the gain at the Gregorian focus.

\subsubsection{Finite Element Model (FEM) correction for non-homologous deformation}

Due to the off-axis design of the GBT, it is not fully homologous. A finite element (FE) model is used to predict the displacements of the primary mirror backup structure as a function of elevation relative to its rigging angle position. These displacements are then used to calculate both the "best-fit" paraboloid, and the residuals from this paraboloid. These residuals are stored in a look-up table, and applied to the antenna via the actuators as the telescope tips in elevation. As an indication of the magnitude of this correction, the rms of the FE model actuator corrections at $15^{\circ}$ elevation is $880 \mu \mathrm{m}$.

Together, the actuator zero-point corrections for construction misalignments, the subreflector focus-tracking to accomodate the gross deformations due to the homologous design, and the FE model derived non-homology corrections allow operation of the antenna at wavelengths as short as $1.5 \mathrm{~cm}$ (frequencies up to $20 \mathrm{GHz}$ ).

\subsubsection{Residual non-homology, thermal deformations and large-scale setting error at the rigging angle}

After applying all of the above corrections, it was apparent from observations at even shorter wavelengths $(7 \mathrm{~mm}$, or $43 \mathrm{GHz})$ that there remained two further large-scale surface error contributions. 1) Residual non-homology, or unmodelled gravitational errors. Despite application of the FE model, the gain-elevation curve continued to show a systematic and repeatable fall-off in gain to high and low elevations, implying that the FE model was not accurately capturing the residual gravitational deformations. (This is not surprising, as we suspect from independent comparisons that the FE model is systematically in error.) The most likely reason for this error is that the FE model has not been yet updated for changes to the telescope structure and weight distribution during construction and operations. 2) Thermal deformations. Observations during daytimes demonstrated that thermal gradients could induce deformations of the antenna, and a resulting loss of efficiency, which could easily exceed the loss of efficiency due to un-modeled gravitational deformations.

Finally, and not obvious from efficiency measurements alone, our OOF investigations revealed that even at the rigging angle under benign thermal conditions, there appeared to be a static, large-scale surface error.

All three of these error components are amenable to investigation, and correction, via the OOF technique.

\section{Measurement strategy}

For all of the measurements presented here, the data consisted of three (total-power) beam-maps taken consecutively, the first with the telescope in-focus and the other two with the telescope $\pm 35 \mathrm{~mm}$ (five wavelengths) out of focus. The de-focusing of the telescope was carried out by moving the sub-reflector in its " $Y$ " direction, that is, in the direction along which its motion does not change the centroid of the beam on the sky. The convention used at the GBT is that a positive displacement corresponds to moving the sub-reflector away from the primary reflector.

The receiver used for these observations was the facility $Q$-band receiver operating at $43.1 \mathrm{GHz}$ and the bandwidth employed was either $80 \mathrm{MHz}$ or $320 \mathrm{MHz}$, depending on the date of observation. This receiver consists of two feeds, each with two polarizations, and thus has two beams on the sky, separated 
by $58 \operatorname{arcsec}(\sim 3.5 F W H M)$ in the azimuth direction. To minimise the effect of sky-brightness variations on the measured beam-maps, the output of these two receivers was differenced so that the effective response of the telescope was the real beam convolved by two delta functions separated by 58 arcsec in the azimuth direction. The aberrations due to both of the feeds being off (and on opposite sides of) the optical axis are negligible in this instance and were not taken into account in the further analysis.

All observations presented here were carried out in continuum mode. The sources used were chosen to be strong (all were stronger than $5 \mathrm{Jy}$ ) and compact with respect to the nominal GBT beam at $43 \mathrm{GHz}$ (16 arcsec); we mainly used the sources J0319+4130 (3C 84), J1256-0547 (3C 279), $\mathrm{J} 2148+0657$, and $\mathrm{J} 2253+1608$ (3C 454.3). Since the system temperature at $Q$-band is typically around $75 \mathrm{~K}$, the theoretical signal to noise ratios expected are in the region of 500:1. Due to gain and atmospheric fluctuations, the actual peak signal to noise ratio we achieved was of the order of 200:1. We have also made successful out-of-focus beam maps using spectral-line observations of strong $\mathrm{SiO}$ masers, although none of these data are used in the analysis presented later.

The beam maps were obtained using the on-the-fly technique (in the Alt/Az frame), that is, the telescope was continuously in motion and a high sampling rate $(10 \mathrm{~Hz})$ used to avoid excessive smearing in the in-scan direction. The maps consisted of seventeen azimuth rows each 350 arcsec in length and separated by eight arcseconds in the elevation direction. The scanning speed of the telescope was fifty arcseconds per second of time and ten seconds was allowed for turn around at the end of each row; hence, the total time required for each map was just under five minutes and the integration time per Nyquist sample was $0.3 \mathrm{~s}$. The entire trajectory was optimised to avoid jerking the telescope during end-of-row turn around. Additionally, the long length of the rows relative to the beam size allows time for any oscillations of the telescope to damp down before it reaches the source. A sample set of observed beam maps (re-gridded for display purposes) is shown in the top row of Fig. 1.

In practice each set of three beam-maps was preceded by pointing and focus measurements although this is not strictly necessary.

\section{Analysis strategy}

The raw data recorded by the telescope needed some minor processing before being analysed with the OOF software. This consisted of calibrating (using the noise-injection diodes), differencing the signals from the two feeds, and removing residual sky brightness variations by fitting linear baselines to the ends of each row and subtracting these baselines from the recorded data.

The resulting time series data consists of antenna offsets (relative to the position of the source) in the azimuth and elevation directions and the antenna temperature for each sample. The data for a set of three out-of-focus beam maps were then analysed using the custom OOF software to produce a map of the total wavefront errors present in the telescope. For the purposes of OOF analysis it is neither necessary nor desirable to regrid these data onto a regular grid. The reason for this is that the model beams can just as well be interpolated to the observed points while regriding the observed data will inevitably cause some information loss.

The technique for analysing beam maps to infer the aberrations is described in detail by Nikolic et al. (2007). In that paper we considered a classical Cassegrain telescope design. The GBT has an off-axis Gregorian design, and so the phase change across the aperture plane induced by moving the secondary reflector is different from the Cassegrain case, and in fact not circularly symmetric, as can be seen from the asymmetry in the elevation direction of the observed beam maps in Fig. 1. But, as long as the correct expression for the phase change due to defocus is used when calculating the model beams, this difference does not affect the performance of the OOF technique in any way.

In the present study we parametrise the possible aberrations as a linear combination of the first five radial orders of Zernike's circle polynomials (see e.g., Born \& Wolf 1970), which we label as:

$Z_{n, l}(r, \theta)= \begin{cases}R_{n, l}(r) * \cos (l \theta) & l \geq 0 \\ R_{n, l}(r) * \sin (-l \theta) & l<0 .\end{cases}$

Here, $R_{n, l}(r)$ is the radial dependence of the Zernike polynomial as given by Born \& Wolf (1970) and $\theta$ is the angle measured from the cross-elevation (left-right in the plots below) direction. Since there are $(n+1)(n+2) / 2$ polynomials up to and including radial order $n$, this gives a total of 18 non-trivial polynomials (the polynomials corresponding to piston and tilts being considered trivial in this instance) which form the basis vectors for the space searched by the optimisation algorithm. The aperture plane amplitude was described as a well-centred, circular Gaussian with a width corresponding to an edge taper of $14.5 \mathrm{~dB}$, close to the design value of the receiver we used. Our initial investigations did not show evidence for significant deviation of these illumination parameters so they were fixed at the above values when fitting for the aberrations shown below. There were only two further free parameters: the overall amplitude of the illumination and the relative gains of the two feeds.

In summary, the free parameters supplied to the minimisation procedure were the 18 non-trivial coefficients of Zernike polynomials, the two tilt terms (to account for the possibility of an error in the telescope pointing), the relative gains of the beams and the overall amplitude.

\section{Validation, repeatability and closure}

We have performed extensive tests to confirm the accuracy and repeatability of this measurement technique. Firstly, we have deliberately introduced a known aberration into the telescope optics, and confirmed that we can correctly measure this. Secondly, we have performed a number of repeat OOF measurements, applying the results of the first before performing the second, and confirming that the surface has actually improved. Finally, we have confirmed the success of the applied correction by independent astronomical measurements. Each of these is discussed in detail below.

\subsection{Retrieval of a known deformation}

In this experiment, we used the active surface system of the GBT to introduce a known "bump" into the figure of the primary reflector. The peak of the bump was $770 \mu \mathrm{m}$. The bump was decomposed into a linear combination of the first five orders of Zernike polynomials and applied by specifying the coefficients to the active surface control system. The procedure for applying the coefficients is exactly the same as that which is used to make corrections to the surface from results of OOF holography, and so this experiment, in addition to testing the ability of the OOF technique to measure aberrations, also provided a useful end-toend test of the system, including coordinate system conventions, Zernike coefficient ordering and so on. 


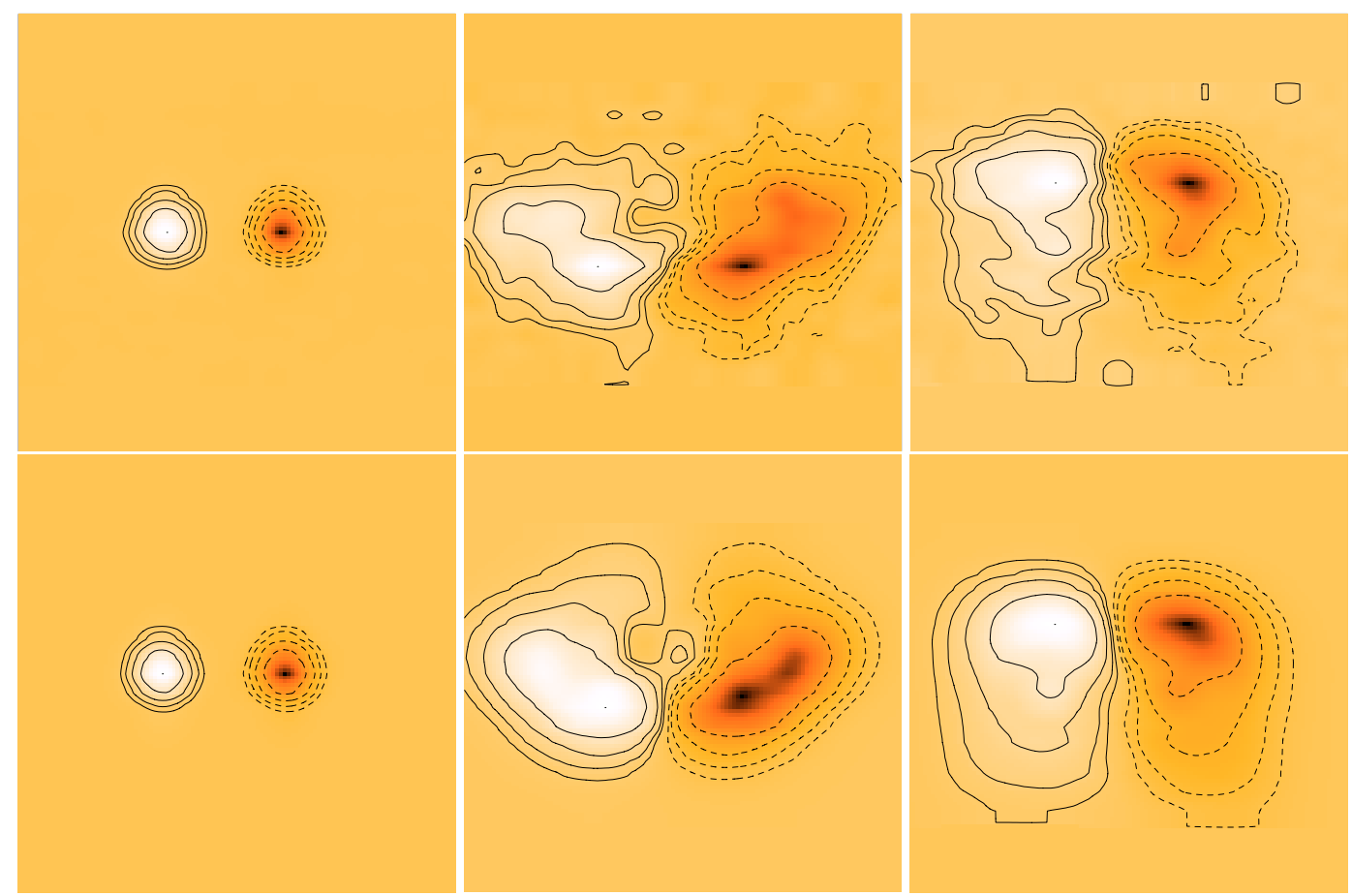

Fig. 1. Top row: a sample set of $43 \mathrm{GHz}$ out-of-focus maps taken on 11th April 2005 using $3 \mathrm{C} 279$ as the source. The left-most map is in-focus, the other two are both $5 \lambda$ out of focus, with the sense of the defocus such that in the centre map the subreflector is further away from the primary than the optimal focus and in the right-most map the subreflector is closer to the primary than optimal. Bottom row: simulated beam maps of the best-fitting model (using Zernike polynomials up to fifth radial order inclusive) to the observed maps in the top row. All of the maps show an area of $210^{\prime \prime} \times 210^{\prime \prime}$ and have the elevation direction running vertically and azimuth direction horizontally. The contours are drawn at logarithmic intervals.

The test consisted of three sets of OOF measurements: the measurement with the bump applied to the surface; and two measurements, proceeding and following the bump measurement, with the nominal surface. The mean of these nominal-surface measurements was used to estimate and remove aberrations not due to the introduced bump. The results are shown in Fig. 2. The top panel shows the wavefront error expected from the bump introduced onto the surface. The bottom panel shows the OOF measurement of wavefront error introduced by the bump, i.e., it is the aperture phase distribution retrieved from beam maps with the bump applied minus the mean aperture phase distribution retrieved from measurements without the bump.

As can be seen from the figure, the measured and expected phase distributions are in fair agreement. As is typical for OOF holography measurements, the largest errors in the retrieved phase distribution are at edges of the dish, which are less well constrained due to the tapered illumination pattern of the astronomical receiver. The illumination-weighted half-path rms difference between the bump and no-bump measurements - i.e., the value which can be used in the Ruze (1966) formula to calculate the expected decrease in aperture efficiency due to residual largescale structure - is $130 \mu \mathrm{m}$; this error is due to the combination of the intrinsic error of the technique and the error in the estimate of aberrations present with no bump applied to the surface.

\subsection{Repeatability, closure and comparison with independent measurements}

Another way to investigate the practical accuracy of the OOF holography technique is to measure the best closure that can be achieved using the technique. This involves observing a set of OOF beam maps and analysing them to obtain the aberrations, compensating for these aberrations by adjusting the primary surface and then repeating the beam map measurement to evaluate how well the initially observed aberrations were compensated for. Because the GBT primary surface is "active", it can be easily and essentially instantaneously adjusted, making it possible to observe both of the sets of beam maps within about one hour.

There are some obvious sources of error with this approach, most notably that the actual aberrations are likely to be changing continuously with time and so correcting for the aberrations at the time of the first measurement can not be expected to fully remove the aberrations at the time of the validation measurement. Nevertheless, a measurement of the closure does provide a good upper limit on the random errors associated with the OOF technique. As this is essentially a self-consistency measurement, it provides no constraint on systematic errors that may be present in the technique. However, independent measurements (such as for example a measurement of the aperture-efficiency of the telescope) can confirm whether the expected performance improvement has been realised.

We have performed a number of measurements of this type. A particularly dramatic example is shown in Fig. 3. In this case, the initial measurement was made at 11 am local time on 11th April 2005, a bright sunny day. The initially measured large scale aberrations (top panel of Fig. 3) were, because of the time of day and resulting thermal deformations, rather large; they correspond to an illumination-weighted, half-path rms wavefront error of around $330 \mu \mathrm{m}$. These OOF measurements were processed and applied to the telescope approximately an hour later, and another set of OOF maps with these corrections applied was taken. The bottom panel of Fig. 3 shows the aberrations derived from this second set of OOF maps, that is the residual aberrations after correcting for what we measured in the top panel. 

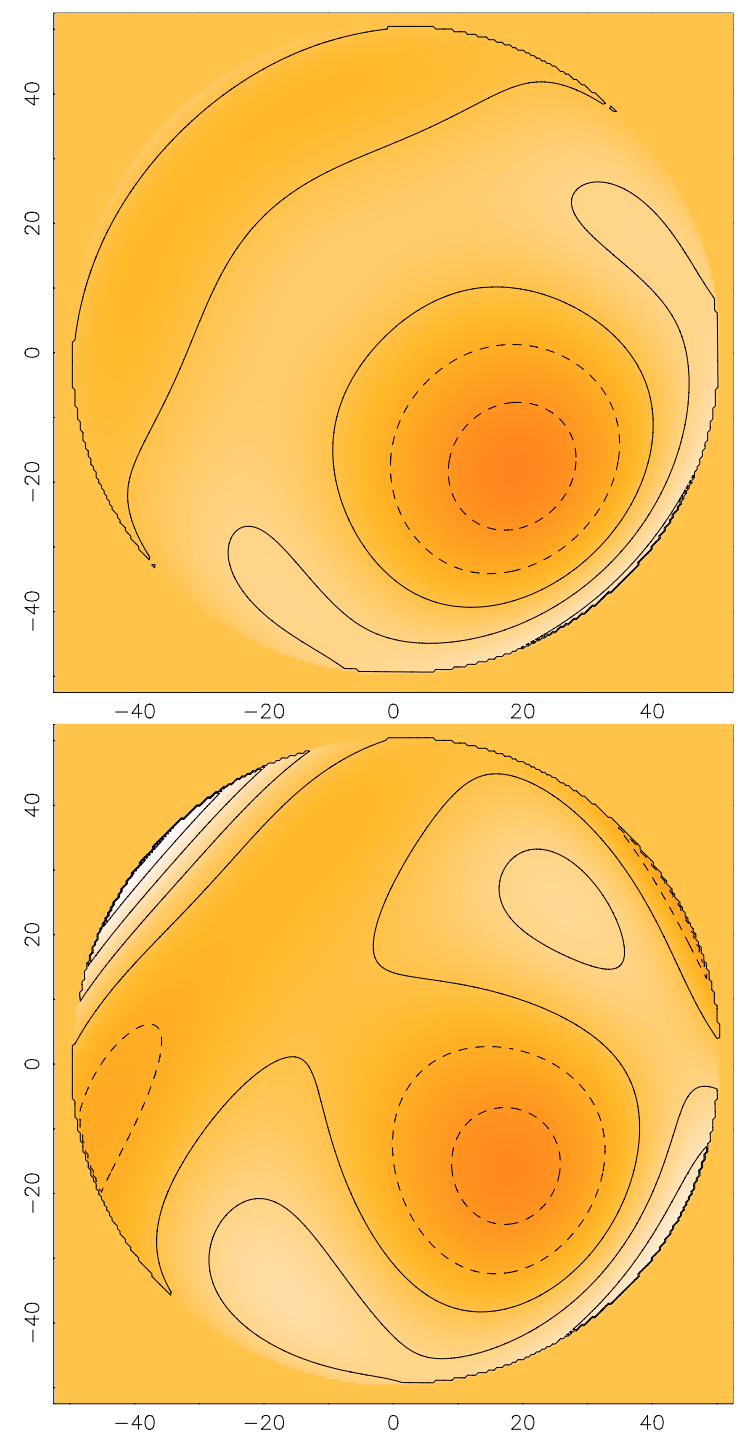

Fig. 2. Test of the OOF technique by measurement of a known wavefront error. Top panel: wavefront error introduced by changing the figure of the primary reflector. Bottom panel: estimate of this wavefront error using the OOF holography technique. The wavefront error is in radians of phase (one radian corresponds to a surface displacement $550 \mu \mathrm{m}$ in the normal direction) and contours are drawn at half-radian intervals. Grayscale is \pm 2 radians.

The estimated weighted half-path rms derived from this second set of OOF maps is $220 \mu \mathrm{m}$.

This particular closure experiment was not ideal due to the significant time delay between the two measurements, which means that the actual thermal and gravitational deformations of the telescope would have changed between them. However, it was chosen to illustrate both the real improvement that this technique can provide, and that it can be used to correct wavefront errors which are clearly dominated by thermal deformations.

Under more ideal (benign night-time) conditions, with the second measurement made within $30 \mathrm{~min}$ of the first, typical half-path rms residuals of $100 \mu \mathrm{m}$ or less were obtained. This residual reflects the random error associated with two OOF measurements, and so is a factor of $\sqrt{2}$ larger than the error of single measurement, which is therefore around $70 \mu \mathrm{m}$ illuminationweighted half-path rms when operating at a wavelength of $7 \mathrm{~mm}$ and using a basis set consisting of the first five radial orders of Zernike polynomials. In terms of the observing wavelength,
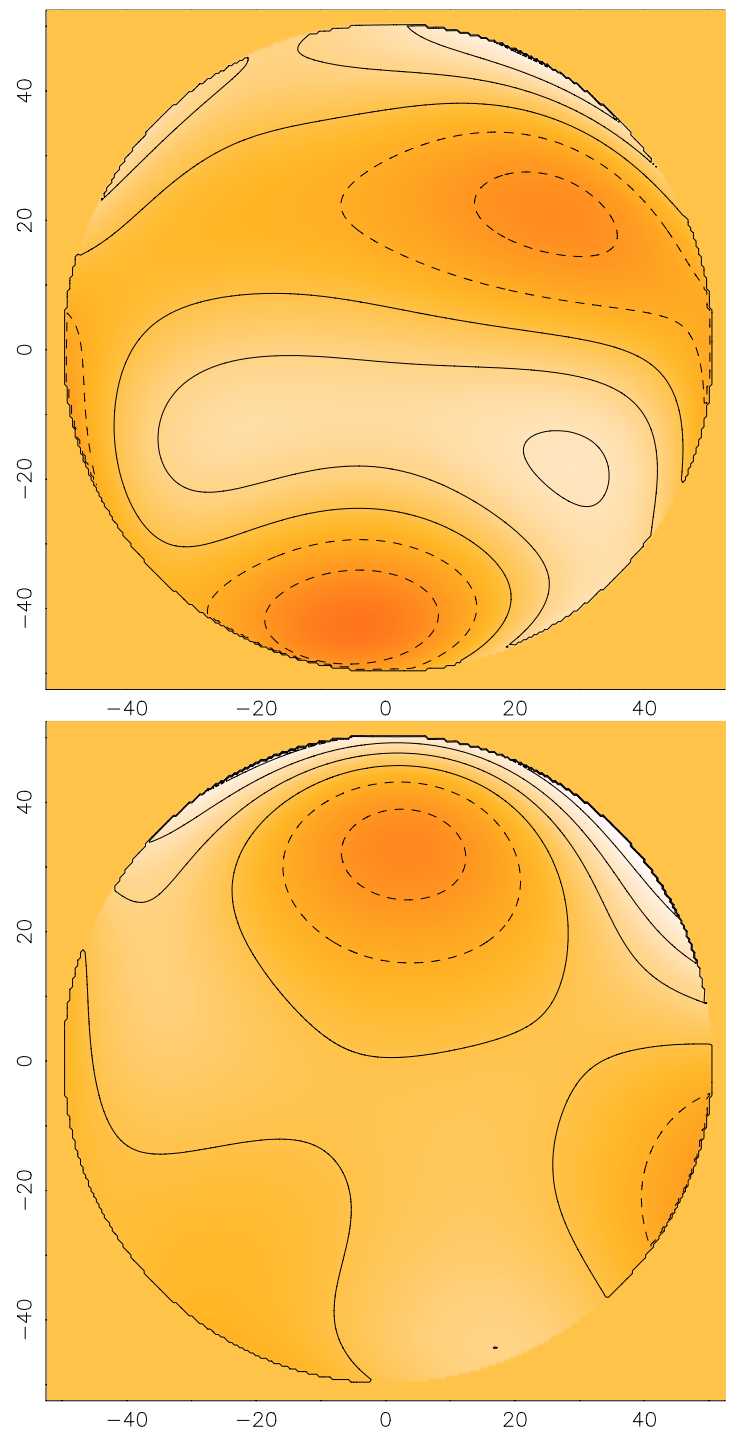

Fig. 3. Closure of the OOF technique at the GBT. Top: wavefront errors derived from daytime OOF measurements; the corresponding illumination-weighted half-path surface rms is $330 \mu \mathrm{m}$. Bottom: the measured wavefront error distribution after applying corrections derived from the measurement shown in the top panel, obtained one hour earlier. The weighted rms is reduced to $220 \mu \mathrm{m}$. Contours in both maps are at half-radian intervals and the grayscale range is \pm 2 radians.

the accuracy of the technique (at the scales being probed) is around $\lambda / 100$.

Finally, as an independent, and for astronomical observing most important, measure of the successful application of the technique we have performed scans across calibration sources in the azimuth and elevation directions before and after applying corrections from OOF observations. For the daytime example given above, the results of such elevation scans are shown in Fig. 4. The improvement is dramatic: the peak antenna temperature (i.e., the on-axis gain of the antenna) was increased by $53 \%$ by making adjustments from the OOF measurement. The sidelobes clearly visible in the scan without any adjustments (top of Fig. 4) have disappeared.

\section{Gravitational deformations}

One of the main advantages of the OOF holography technique is of course that it allows measurements to be made over the full 

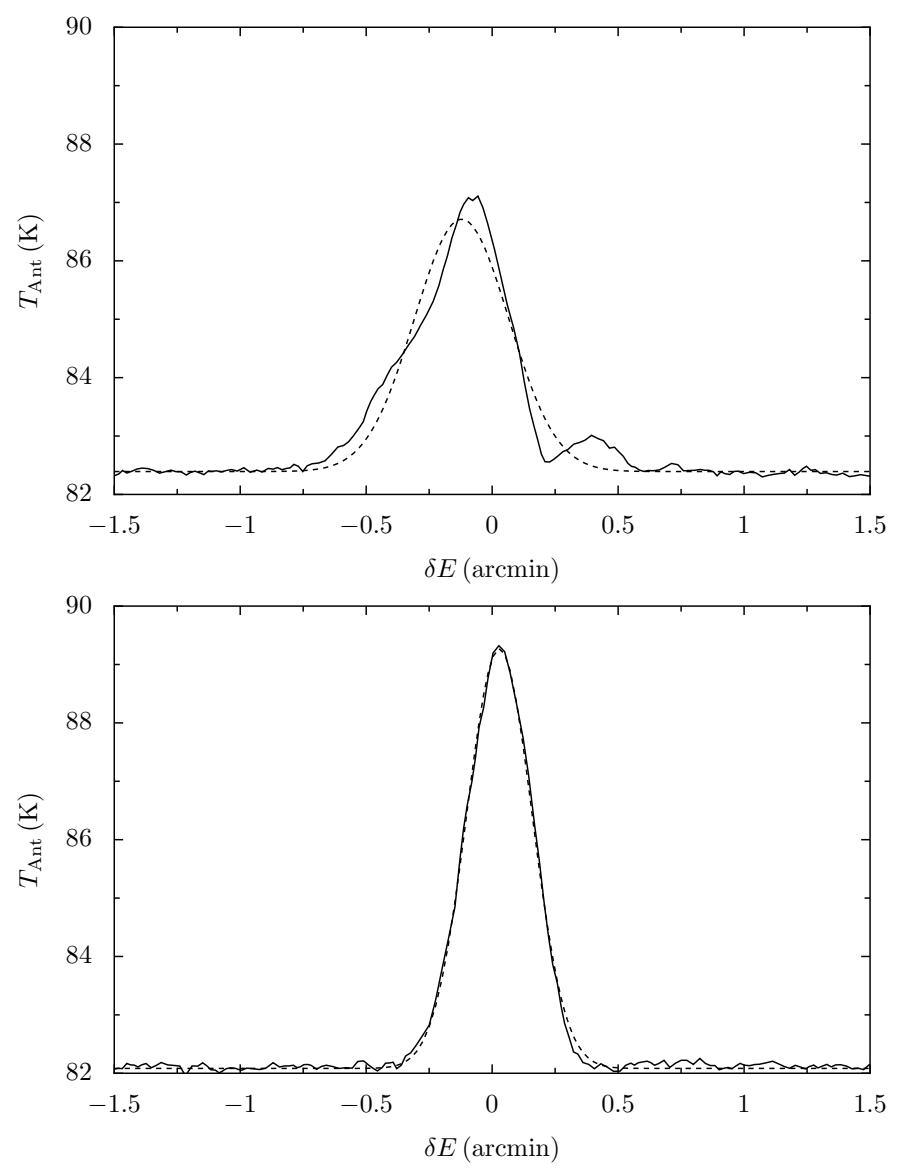

Fig. 4. Elevation scans through a calibrator source before (top) and after (bottom) applying corrections derived from OOF measurements. Dashed lines show best-fitting Gaussian models to the scans.

range of elevations at which astronomical observations are normally carried out. This makes it possible to measure the aberrations which are due to gravitational deformation of the telescope but are not fully corrected for by the active surface and the standard finite-element model. Such aberrations can then be corrected by operating the active surface using the sum of the FE model and an empirical model derived from the measurements.

In order to measure these gravitationally-induced aberrations, we carried out a campaign of 36 OOF observations over three nights in the Spring and Autumn of 2005. The FE model was applied through the active surface as normal for these observations and so we were measuring only the residual deformation. In order to minimise the effect of thermal gradients in the telescope structure, the observations were carried out only between approximately midnight and sunrise of each night; measurements during the day or early evening show that aberrations due to thermal effects are comparable to or exceed that due to uncompensated gravity deformation. After analysis these data produced 36 sets of coefficients of Zernike polynomials describing the wavefront errors over a wide range of elevations.

In order to examine if the OOF measurements support the hypothesis that the existing FE model does not fully correct for gravitational deformation of the GBT, we first computed the expected effect of the large scale errors on the telescope gain. This is summarised in Fig. 5, which shows the expected decrease in on-axis gain due to the large-scale structure derived from each OOF measurement plotted against elevation at which the observation was made. The efficiency values have an estimated

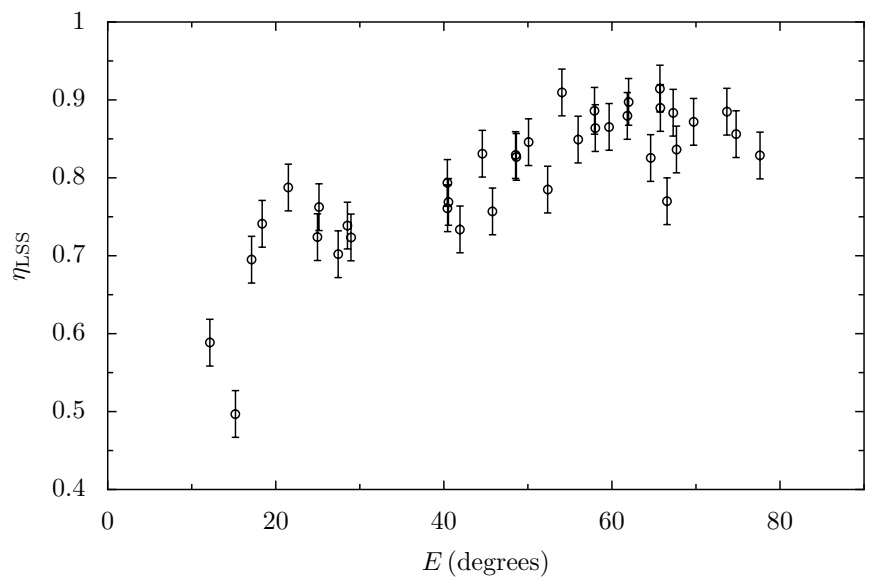

Fig. 5. Predicted decrease of the on-axis gain at $43 \mathrm{GHz}$ due to measured large-scale structure $\left(\eta_{\mathrm{LSS}}\right)$ derived from OOF measurements over a range of elevations $(E)$.

random error of $3 \%$, corresponding to the $100 \mu \mathrm{m}$ weighted $\mathrm{rms}$ error of the wavefront maps. It can be seen from the plot that the large-scale wavefront errors level off around the rigging angle of 50.3 degrees but they become increasingly important toward low elevations. This is consistent with gravitational deformation. It is interesting that we measure a modest amount $(\approx 150 \mu \mathrm{m} \mathrm{rms})$ of large-scale structure even at rigging angle, implying there is a systematic error in the actuator zero-point settings. Correction of this large-scale error produces a modest $(\approx 10 \%)$ increase in efficiency at $43 \mathrm{GHz}$.

After the first night of OOF measurements, we made a simple model to correct for these gravitational deformations by linearly interpolating the coefficients as a function of elevation between the OOF models. This model was tested by measuring the aperture efficiency of the GBT as a function of elevation with only the FE model and the finite-element plus correction derived from OOF measurement model (FEM+OOF) applied. The aperture efficiencies were measured by using strong point sources which we bootstrapped from a primary flux calibrator. These measurements were made in total power mode, by interleaving measurements of empty sky and source, and using the same frequency and receiver as used for holography. The results are shown in Fig. 6.

Unfortunately, the aperture efficiency measurements suffer from significant uncertainties due to fluctuations in atmospheric opacity, as well as pointing and tracking errors of the telescope which are not negligible when observing at $7 \mathrm{~mm}$. Nevertheless, it is quite clear from the results shown that there is a measurable improvement to the aperture efficiency at low elevation. Furthermore, when the FEM+OOF model is applied, there does not appear to be significant remaining variation of aperture efficiency with below 70 degrees elevation. The apparent fall-off at higher elevations may be due to effects not associated with gravitational deformation, such as poorer tracking performance of the telescope.

Using data from all three nights, we continued to investigate if the shape of the measured deformations is consistent with the effects of gravity. As described above, the result of each individual measurement is a set of coefficients of Zernike polynomials. Working on the assumption that the coefficients are completely independent (this is only approximately true, see Nikolic et al. 2007) we considered each one separately and found the best-fitting function of the form expected for gravitational deformations. If the telescope is a linear elastic structure that is 

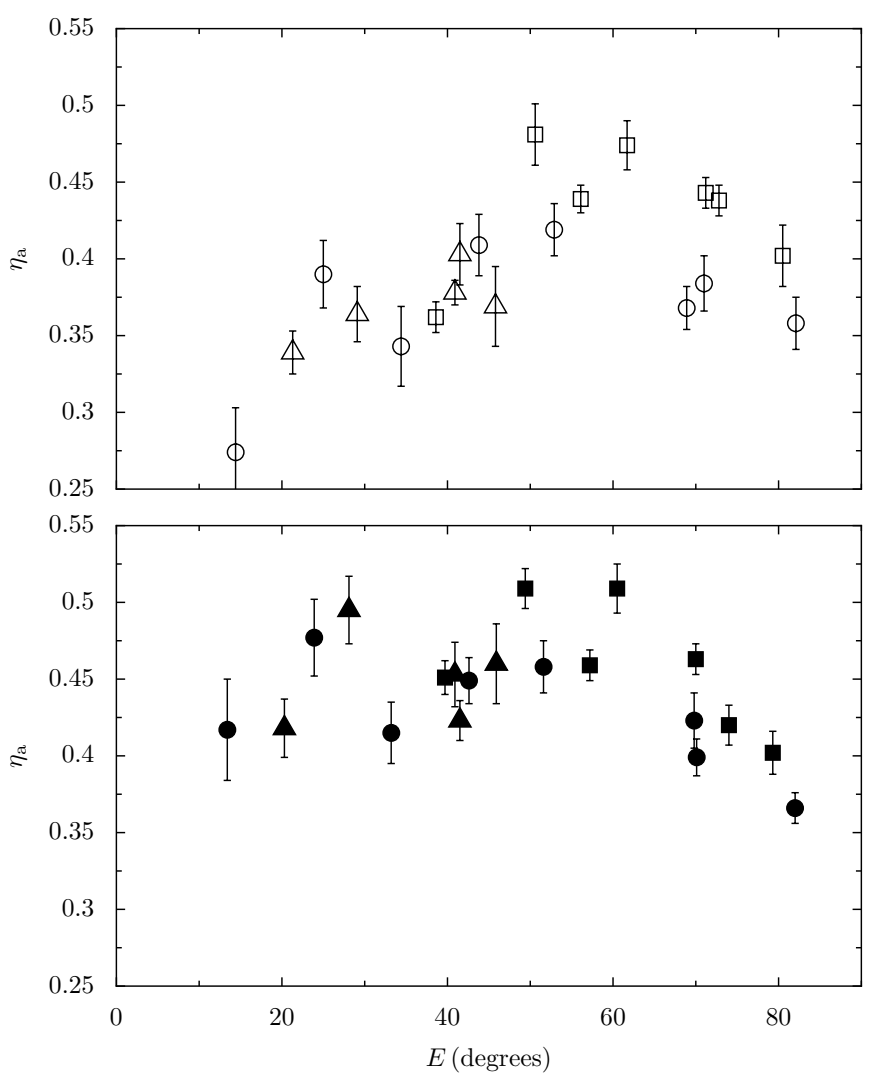

Fig. 6. Aperture efficiency at $43 \mathrm{GHz}\left(\eta_{\mathrm{a}}\right)$ vs. elevation $(E)$ measurements on 11 April 2005. Top panel, with open symbols, shows the measurements made with the nominal FE model; bottom panel, with filled symbols, shows the measurements with the FEM+OOF model applied. Sources used for the efficiency measurements are denoted by symbol types: 1642+3948 (square), 1256-0547 (triangle) and 3C 286 (circle).

symmetric in the plane of the feed arm, then gravitationally induced aberrations should depend on the two resolved components of gravity only, that is, $g \sin (E)$ and $g \cos (E)$, where $E$ is the elevation of the telescope. Taking into account the possibility of a further, gravitationally-independent, setting error, the coefficients of Zernike polynomials, $z_{i}$, should follow the functional form:

$z_{i}(E)=a \sin (E)+b \cos (E)+c$.

This then was the function which was fitted to the observed coefficients, with all $a, b$ and $c$ being free parameters. The observed data and the best-fitting functions of the form in Eq. (2) are shown in Fig. 7. As it can be seen in the figure, the measured values of many of the coefficients have an elevation dependence that is clearly consistent with gravitational deformation. The coefficients which show little trend with elevation (e.g., $n=3, l=1$ and $n=3, l=3$ ) tend to correspond to Zernike polynomials that are strongly asymmetric in the cross-elevation direction.

Within the uncertainties caused by other effects, as expected the gravitational deformations appear completely repeatable. The full linear elastic structure model described above has been incorporated into the GBT active surface control system, and is now in routine operation.

\section{Discussion}

We begin by re-iterating that the OOF technique measures only wavefront errors which are correlated over large distances in the aperture plane. In this study we described surface errors as a linear combination of 18 Zernike polynomials. Larger beam maps with better signal to noise ratios (as may be possible to obtain using focal plane arrays) will provide opportunities to increase the size of the basis set used in the fitting procedure, but it is unlikely it will be possible to use this technique to set the 2000 individual panels of the GBT. The OOF technique is nevertheless useful because effects such as gravitational and thermal deformations, and mis-alignment of the optical elements, naturally induce large-scale wavefront errors.

In the first experiment, described in Sect. 4.1, we demonstrate the ability of the OOF technique to measure deformations of the telescope surface. The combination of relatively short measurement time and the GBT active surface system allowed us to introduce a realistic sized-bump to the primary reflector (the bump peak was around $700 \mu \mathrm{m}$ or $\lambda / 10$ ) and the surface map retrieved using the OOF technique is clearly very similar to the applied bump. The illumination weighted rms error of the bump measurement was $130 \mu \mathrm{m}$. As described in Sect. 4.2, when no large deformation is applied to the surface, the random accuracy of the OOF technique can be as low as $70 \mu \mathrm{m}$ (i.e., $\lambda / 100$ ). If OOF measurements are made at the shortest wavelengths at which astronomical observing are to be carried out - which should normally be possible because only the standard astronomical receivers are used - this accuracy should be more than adequate for practical application.

We then investigated the practical applications of these measurements of wavefront errors. Our main target was gravitational deformations because, as they depend on elevation only they are expected to be repeatable, and because the variation of the GBT gain with elevation suggests that they are present even when the FE model is used. We used a series of OOF measurements to derive an elevation-dependent correction to the FE model, and with these corrections applied to the telescope, we find that the measured aperture efficiency is largerly independent of elevation (filled symbols in Fig. 6). This suggests that, as expected, the major aberrations induced by gravity are large-scale and measurable using the OOF technique, and, that they can be successfully corrected. We note that such measurements and a model for gravitational deformation may be useful even for telescopes without an active surface. For example, if the panels of the primary surface are set using holography with a ground-based transmitter, i.e., essentially at zero degree elevation, it would normally be desirable to make an adjustment to this setting so that the expected gravitational deformations are as small as possible at higher elevation, close to the elevations used for typical observations. Such an adjustment could easily be determined using the technique described here.

We also investigated measurement and correction of aberrations due to thermal effects on the telescope structure. These thermal deformations of the GBT optics can easily dominate the total wavefront error during the daytime as evidenced by degradation of the aperture efficiency and beam shape of the GBT after sunrise. In Sect. 4.2 we showed that corrections derived from $\mathrm{OOF}$ measurements during the daytime can dramatically improve both the gain and side-lobe structure of the telescope. This demonstrates that the OOF technique described here is fully capable of measuring and correcting for thermal effects. Subsequent daytime measurements have confirmed, as expected, that the deformations due to these effects are large-scale, and smoothly varying with time. Unfortunately, although thermal effects are expected to be fully repeatable in the sense that the same thermal environment should produce the same deformation; the varying thermal conditions, and orientation history of 

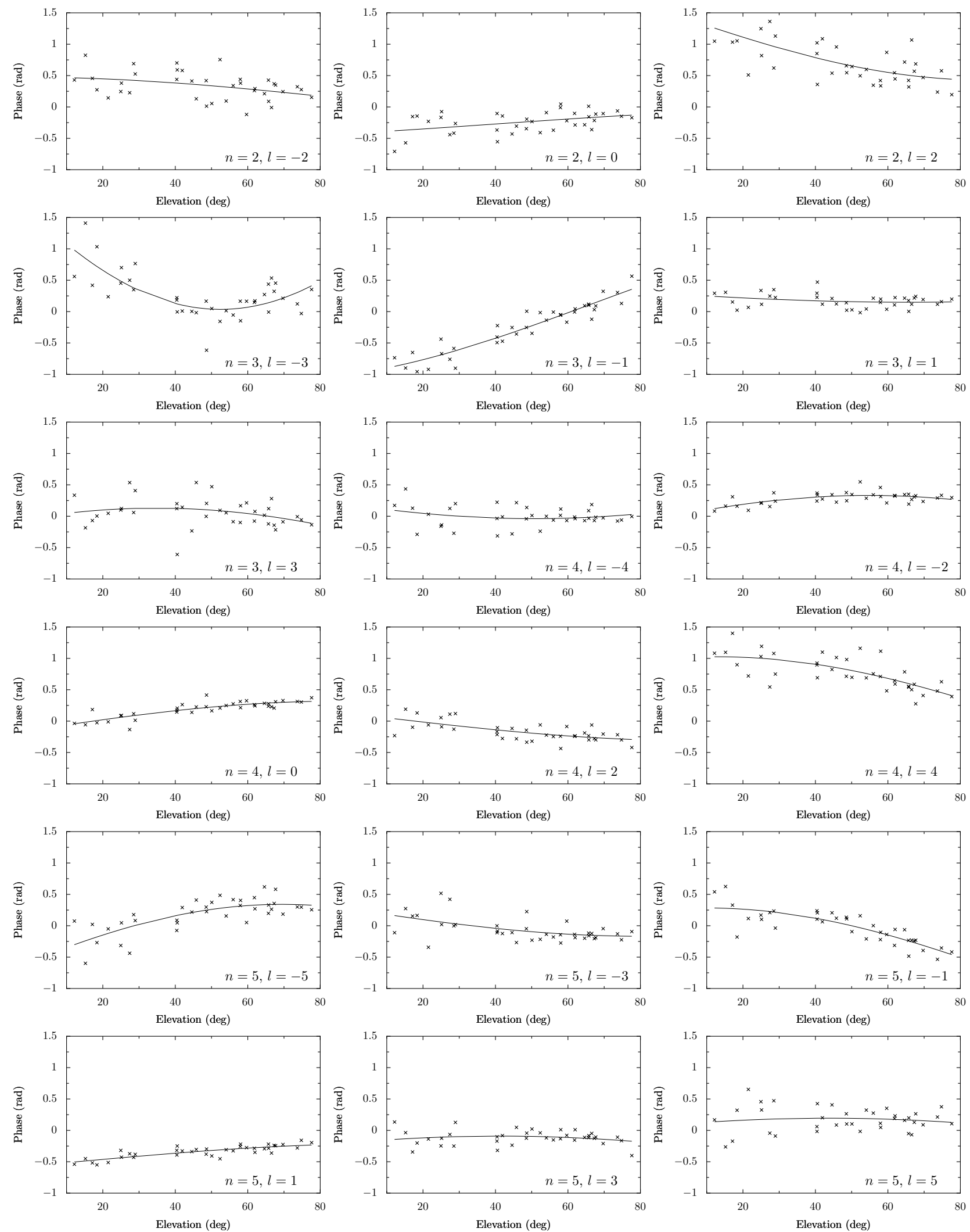

Fig. 7. Measured values of the coefficients of Zernike polynomials as a function of elevation (crosses) and the best fitting model of the form shown in Eq. (2) (solid line). Units throughout are radians of phase at the aperture edge (one radian of phase corresponds to a surface displacement $550 \mu \mathrm{m}$ in the normal direction), $n$ is the radial and $l$ is the angular order of the polynomial. 
the telescope from day to day means that there is no single parameter which can be used to parameterise the deformations, analagous to the telescope elevation for the gravitational deformations.

The time it currently takes to obtain an OOF data-set with our dual-pixel receiver means that it is impractical either to attempt to characterise the entire range of thermal environments with OOF maps, or to perform routine measurements and adjustments in "real time". The results shown are, however, a proof of principle that one of these approaches (most likely the real time correction) will be useful once multi-pixel focal-plane arrays become available. With focal-plane arrays, the time required to make the beam maps will not be much longer than required for optimising the focus of the telescope. In this case, the OOF analysis used here may be used to correct the focus and higher order aberrations whenever a traditional focusing measurement would have been done.

\section{Summary}

We have performed "OOF" phase-retrieval holography measurements of the 100-metre Green Bank Telescope, using the technique described by Nikolic et al. (2007). The advantages of the OOF technique which motivated us to carry out these observations are:

1. The measurements provide a map of total large-scale wavefront errors (aberrations) present in the system.

2. The aberrations can be measured over the full range of elevations making it possible to infer the effects of gravity on the telescope.

3. The time required for each measurement is relatively short.

4. The covenience of being able to perform the measurements using the standard astronomical receivers, with no special hardware or software setup, brings considerable operational advantages.

In addition, and very importantly, the active surface of the GBT has allowed us to immediately and easily test our measurements. As far as general practicalities of using the OOF technique are concerned:

1. We have demonstrated that OOF holography with astronomical sources and receivers can be routinely carried out on a large millimeter-wave radio telescope. A full set of observations takes of the order of $25 \mathrm{~min}$.

2. Observing at a wavelength of $7 \mathrm{~mm}$, using a measurecorrect-measure cycle, we measure an illumination-weighted half-path large-scale error of around $100 \mu \mathrm{m}$, indicating that the random error associated with the OOF technique in this case is $\approx \lambda / 100$.
3. Correcting for the measured aberrations produces an improvement in the aperture efficiency close to the expected improvement.

More specifically to the GBT, we found:

1. At night time and at elevations close to the rigging angle, there is a small but measurable large-scale wavefront error.

2. By making OOF maps at a number of elevations we have been able to derive a model for aberrations which is consistent with gravitational deformations. This model, which is essentially a small refinement to the existing finite element model for the telescope, is now in routine use for highfrequency observing.

3. Applying this model makes an improvement to the aperture efficiency at elevations smaller than 40 degrees, largely making the aperture efficiency independent of elevation. This indicates that the deformations induced by gravity are largescale and measurable using this technique.

4. Daytime thermal deformations of the GBT are also large scale, and smoothly varying with time. The time currently required to obtain an OOF dataset makes attempts to correct for thermal deformations impractical at this time. However, this technique should become directly applicable once array receviers are available on the telescope.

Acknowledgements. We would like to thank the referee for his comments which significantly improved this paper.

The National Radio Astronomy Observatory is a facility of the National Science Foundation operated under cooperative agreement by Associated Universities, Inc. B. Nikolic would like to thank NRAO for the appointment to a post-doctoral position during the course of which the work described here was carried out. We acknowledge George Moellenbroack for assistance with data pre-processing software in the early stages of this project.

Although we have carried out the investigations described here, the GBT active surface itself, and the associated control software, is the result of many years of hard work by numerous past and present members of the NRAO staff. We applaud them for providing us with such a wonderful system to work with.

\section{References}

Anderson, A. P., \& Sali, S. 1985, IEEE Proc., 132, 291

Born, \& Wolf 1970, Principles of Optics (Oxford: Pergamon) Jewell, P. R., \& Prestage, R. M. 2004, in Proc. SPIE, 5489, 312 Lacasse, R. J. 1998, in Proc. SPIE, 3351, ed. H. Lewis, 310

Morris, D. 1985, IEEE Transactions on Antennas and Propagation, AP-33, 74 Nikolic, B., Hills, R., \& Richer, J. 2007, A\&A, 465, 679

Ruze, J. 1966, Proc. IEEE, 54, 663

von Hoerner, S. 1967, AJ, 72, 35 